\title{
Overlapping and non-overlapping roles of the class-I histone deacetylase-1 corepressors LET-418, SIN-3, and SPR-1 in Caenorhabditis elegans embryonic development
}

\author{
Yukihiro Kubota ${ }^{1} \cdot$ Yuto Ohnishi ${ }^{2} \cdot$ Tasuku Hamasaki $^{2} \cdot$ Gen Yasui $^{2} \cdot$ Natsumi Ota $^{2} \cdot$ Hiromu Kitagawa $^{2} \cdot$ Arashi Esaki $^{2}$. \\ Muhamad Fahmi ${ }^{2} \cdot$ Masahiro Ito ${ }^{1,2}$
}

Received: 16 December 2020 / Accepted: 1 March 2021 / Published online: 19 March 2021

(c) The Author(s) 2021

\begin{abstract}
Background Histone deacetylase (HDAC)-1, a Class-I HDAC family member, forms three types of complexes, the nucleosome remodeling deacetylase, Sin3, and CoREST complexes with the specific corepressor components chromodomainhelicase-DNA-binding protein 3 (Mi2/CHD-3), Sin3, and REST corepressor 1 (RCOR1), respectively, in humans.

Objective To elucidate the functional relationships among the three transcriptional corepressors during embryogenesis. Methods The activities of HDA-1, LET-418, SIN-3, and SPR-1, the homologs of HDAC-1, Mi2, Sin3, and RCOR1 in Caenorhabditis elegans during embryogenesis were investigated through measurement of relative mRNA expression levels and embryonic lethality given either gene knockdown or deletion. Additionally, the terminal phenotypes of each knockdown and mutant embryo were observed using a differential-interference contrast microscope. Finally, the functional relationships among the three corepressors were examined through genetic interactions and transcriptome analyses.

Results Here, we report that each of the corepressors LET-418, SIN-3, and SPR-1 are expressed and have essential roles in C. elegans embryonic development. Our terminal phenotype observations of single mutants further implied that LET-418, SIN-3, and SPR-1 play similar roles in promoting advancement to the middle and late embryonic stages. Combined analysis of genetic interactions and gene ontology of these corepressors indicate a prominent overlapping role among SIN-3, SPR-1, and LET-418 and between SIN-3 and SPR-1.

Conclusion Our findings suggest that the class-I HDAC-1 corepressors LET-418, SIN-3, and SPR-1 may cooperatively regulate the expression levels of some genes during C. elegans embryogenesis or may have some similar roles but functioning independently within a specific cell.
\end{abstract}

Keywords C. elegans $\cdot$ Corepressor $\cdot$ Embryo $\cdot$ HDAC $\cdot$ RNA-seq $\cdot$ Comparative transcriptome

Yukihiro Kubota and Yuto Ohnishi contributed equally to this work.

Masahiro Ito

maito@sk.ritsumei.ac.jp

1 Department of Bioinformatics, College of Life Sciences, Ritsumeikan University, 1-1-1 Nojihigashi, Kusatsu, Shiga 525-8577, Japan

2 Advanced Life Sciences Program, Graduate School of Life Sciences, Ritsumeikan University, 1-1-1 Nojihigashi, Kusatsu, Shiga 525-8577, Japan

\section{Introduction}

During embryonic development, daughter cells generated from fertilized eggs contain the same genomic information as the progenitor cells when the cell division process is completed. Although they have identical genome sequences, daughter cells can differentiate from precursor cells within developing tissues and organs through the epigenomic control of gene expression. Therefore, epigenomic modifications play important roles in normal embryonic development (Cavalli 2006). Epigenomic modifications are modulated via chemical changes to histones and DNA. Acetylation and the methylation of histones affect the regulation of gene expression by influencing histone-DNA and histone-protein interactions. Histone modifications are regulated by transferases 
and hydrolases (Cavalli 2006). Histone acetyltransferases promote this process to neutralize the positive charge of the histone tail, thus acting as positive transcriptional regulators by weakening the physical interaction between the histone tail and DNA (Garcia-Ramirez et al. 1995). In contrast, histone deacetylases (HDACs) remove acetyl groups from histones and negatively regulate transcription by enhancing the physical interaction between the histone tail and DNA (Cosgrove et al. 2004).

The 18 human HDAC proteins are divided into four classes based on their sequence homologies with the four yeast HDAC proteins (Vaquero et al. 2007; Yang and Seto 2008). Among four class-I HDACs members, HDAC-1 forms complexes with multiple components, such as transcriptional corepressors and DNA binding proteins, and promote histone deacetylation to suppress the transcription of target genes (Hayakawa and Nakayama 2011). The HDAC-1 transcriptional corepressor forms three types of complexes in humans, the NuRD complex, Sin 3 complex, and CoREST complex, with specific corepressor components (Mi2/CHD3, Sin3, and RCOR1, respectively) (Hayakawa and Nakayama 2011). These complexes are thought to function as transcriptional repressors by inhibiting the transcription of their target genes (Hayakawa and Nakayama 2011). HDACs have been implicated in regulating various vital processes, such as DNA repair, lipid metabolism, cell cycle progression, and the circadian rhythm (Feng et al. 2011; Knutson et al. 2008; Miller et al. 2010; Sun et al. 2012; Wilting et al. 2010). Furthermore, HDAC-1 proteins have been shown to play important roles in the embryogenesis of multiple model organisms (Mannervik and Levine 1999; Montgomery et al. 2007; Shi and Mello 1998; Vecera et al. 2018). However, the mechanism by which the interplay among the three HDAC-1 complexes regulates embryonic development remains unknown.

The nematode Caenorhabditis elegans is a model multicellular organism, for which the whole genome sequence and entire cell lineage have been completely identified (Genome sequence of the nematode $C$. elegans: a platform for investigating biology 1998; Sulston et al. 1983). Therefore, $C$. elegans is a reliable model organism to analyze the regulatory mechanism of embryogenesis. The constituents of the HDAC complex are also conserved in $C$. elegans (Wenzel et al. 2011). Human HDAC-1 shares conserved sequences with $C$. elegans ortholog of HDAC-1, HDA-1. Furthermore, C. elegans $h d a-1$ can help regulate vulval development (Ranawade et al. 2013). LET-418, SIN-3, and SPR-1 are $C$. elegans homologs of the human transcriptional corepressor components Mi-2/CHD3, SIN3, and RCOR1, respectively, and each corepressor has been shown to be involved in both embryonic and post-embryonic development, driving specific functions, such as male sensory cell formation, gonadal morphogenesis, and vulval development (Bender et al. 2007; Beurton et al. 2019; Choy et al. 2007; Käser-Pébernard et al.
2016; Passannante et al. 2010; Saudenova and Wicky 2018; Solari and Ahringer 2000; von Zelewsky et al. 2000). However, the functional relationships among these three transcriptional corepressors in C. elegans embryogenesis remain unexplored.

In this study, we identified functional similarities and differences among the transcriptional corepressors LET-418, SIN-3, and SPR-1 to understand the functional relationships of the related HDAC-1 corepressors during embryogenesis. First, we determined whether hda-1, let-418, sin-3, and spr-1 participate in embryogenesis. Then, we analyzed the genetic interactions between two corepressors to identify relationships among all three corepressors. Finally, comprehensive comparative analysis of the target genes of the LET-418, SIN-3, and SPR-1 complexes was performed via RNA sequencing (RNA-seq). We then combined our analysis of genetic interactions with gene ontology (GO) analysis of these corepressors, which suggests a prominent overlapping role among SIN-3, SPR-1, and LET-418 and between SIN-3 and SPR-1.

\section{Materials and methods}

\section{Caenorhabditis elegans strains}

Caenorhabditis elegans strains were derived from the wildtype (WT) Bristol strain (Brenner 1974). Worms were incubated on nematode growth medium (NGM) and fed OP50 bacteria at $20^{\circ} \mathrm{C}$. When performing RNA-interference (RNAi) experiments, the animals were fed dsRNA-expressing Escherichia coli HT115 (DE3), which were maintained at $20^{\circ} \mathrm{C}$.

Caenorhabditis elegans strains with the following putative null alleles were used for our analysis: $\sin -3(\mathrm{tm} 1276)$ (National BioResource Project, Japan), spr-1(ok2144) (C. elegans Gene Knockout Consortium), and weak loss-offunction allele let-418 (n3536) (Ceol et al. 2006) (Caenorhabditis Genetics Center). To compare the gene functions of these three corepressors under the same conditions, we analyzed let-418(n3536) in a semi-permissive condition at $20{ }^{\circ} \mathrm{C}$.

\section{Sample preparation for RNA-seq}

To isolate synchronized early $C$. elegans embryos, the following four steps were performed. (1) Adult worms (WT and mutant) bearing fertilized eggs were treated with bleach solution, and the eggs were extracted. (2) The eggs were cultured in S-basal until all eggs hatched to synchronize the developmental stage, and subsequently OP50 solution was added to the S-basal. (3) The synchronized worms were incubated until they grew to the young adult stage, capable 
of bearing 2-3 fertilized eggs. (4) The early embryos were isolated by bleaching.

\section{Total RNA extraction}

For RNA-seq and reverse-transcriptase quantitative polymerase chain reaction (RT-qPCR) analyses, total RNA was extracted from the WT, let-418(n3536), sin-3(tm1276), and spr-1(ok2144) strains using the TRI Reagent (Molecular Research Center, Inc., Cincinnati, OH). Following DNA digestion, total RNA was extracted using an RNeasy Mini Kit (Qiagen, Hilden). The extracted RNA was qualitatively evaluated using a Bioanalyzer (Agilent Technologies, Palo Alto, CA) and the Agilent RNA 6000 Nano Kit (Agilent Technologies, Palo Alto, CA).

\section{RT-qPCR analysis}

Complementary DNA (cDNA) was synthesized from total RNA from WT C. elegans at each developmental stage (early-stage embryo, middle-stage embryo, late-stage embryo, first larva, and young adult) using the PrimeScript RT Reagent Kit (Takara, Kusatsu). RT-qPCR was performed in a StepOnePlus ${ }^{\mathrm{TM}}$ qPCR system (Thermo Fisher Scientific, Waltham, MA) using THUNDERBIRD SYBR qPCR Mix (Toyobo, Osaka). The expression levels of $h d a-1$, sin-3, let418 , and $\mathrm{spr}-1$ were normalized to those of a gene encoding an iron binding protein (Y45F10D.4), which was previously characterized as a reference gene because its expression is stable during development in both WT and mutant strains (Hoogewijs et al. 2008). The following primers were used to amplify Y45F10D.1 (Y45F10D.4_F, 5'-GTCGCTTCA AATCAGTTCAGC-3'; Y45F10D.4_R, 5'-GTTCTTGTC AAGTGATCCGACA-3'), $h d a-1$ (hda-1_F, 5'-GGTCAA GGGCACGTCATGAAGCC-3'; hda-1_R, 5'-CTCGTCGCT GTGAAAACGAGTC-3'), let-418 (let-418_F, 5'-GTGCTG CTATCGGATTGACAGACG-3'; let-418_R, 5'-GGGTTT GCCTCCAGTATTTGTGGC-3'), sin-3 (sin-3_F, 5'-GCA ACCGTGGAATTGATGA-3'; sin-3_R, 5'-GTTGATTCG GTGTTGTTCGAC-3'), and spr-1 (spr-1_F, 5'-CTCCAT CTCCATATCCTGAAGC-3'; spr-1_R, 5'-GCACGGCAT TCTGGACGATTCATCG-3').

\section{Feeding RNAi}

RNAi was performed using the feeding RNAi method with freshly prepared RNAi feeding plates, as described previously (Kamath et al. 2001). Full-length hda-1, let-418, sin3 , and spr-1 cDNA was isolated from a C. elegans cDNA library and inserted into the feeding RNAi vector L4440 (Addgene, Cambridge, MA, USA). An L4440 vector lacking an insert was used as a negative control. After confirming that each inserted sequence was correct, the feeding vectors were individually transformed into E. coli HT115(DE3) cells, which were then seeded on NGM agar plates containing Luria-Bertani medium and $50 \mu \mathrm{g} / \mathrm{mL}$ ampicillin and cultured for $12 \mathrm{~h}$. Then, each culture was seeded onto a $60 \mathrm{~mm}$ NGM agar feeding plate containing $50 \mu \mathrm{g} / \mathrm{mL}$ ampicillin and $1 \mathrm{mM}$ isopropyl $\beta$-D-1-thiogalactopyranoside and then incubated at $25^{\circ} \mathrm{C}$ for $8 \mathrm{~h}$. L4-stage worms were transferred onto a feeding plate and cultured at $20^{\circ} \mathrm{C}$. The phenotypes of the F2 embryos were analyzed, except for those fed RNAi bacteria expressing double-stranded $h d a-l$ RNA, which were analyzed in the F1 embryos.

\section{Analysis of embryonic lethality}

To analyze embryonic lethality, fertilized eggs were isolated by dissecting 1-day-old adult worms, after which the fertilized eggs were incubated at $20^{\circ} \mathrm{C}$ for $24 \mathrm{~h}$ and the ratio of the unhatched embryos was scored. To characterize the timing of the terminal phenotype, we defined embryonic lethality in early embryos as those that died before the ventral cleft-enclosure stage. Embryonic lethality in middle embryos was defined as those that died between the ventral cleft-enclosure stage and the comma stage. Embryonic lethality in late embryos was defined as those that died between the 1.5 -fold stage and the threefold stage. To analyze the terminal phenotypes of the dead embryos, Nomarski microscopy was performed using a Zeiss Axio Imager A1 microscope equipped with an EC Plan-Neofluar $40 \times \mathrm{NA}$, 0.75 objective (Zeiss), AxioVision software (Zeiss), and an AxioCam MRc digital camera. The images were processed using Adobe Photoshop CS6.

\section{Statistical analyses of embryonic lethality}

$P$-values (determined using Fisher's exact test) were used to assess the significance of differences observed in terms of embryonic lethality. To analyze the embryonic lethality of let-418(n3536); control(RNAi), let-418(n3536);sin-3(RNAi), let-418(n3536);spr-1(RNAi), sin-3(tm1276);control(RNAi), sin-3(tm1276);let-418(RNAi), sin-3(tm1276);spr-1(RNAi), spr-1(ok2114);control(RNAi), spr-1(ok2114);let-418(RNAi), and spr-1(ok2114);sin-3(RNAi) strains, the numbers of embryonic-lethal embryos and hatched (non-embryoniclethal) embryos were compared.

\section{RNA-seq analysis}

RNA-seq analysis $(\mathrm{N}=3)$ of the WT, sin-3(tm1276), let418(n3536), and spr-1(ok2144) strains was performed using a MiSeq instrument (Illumina, San Diego, CA), following the manufacturer's recommended protocols (available on the Illumina website). Library preparation for RNA-seq was performed using the TruSeq Stranded 
Total RNA LT Sample Prep Kit (Illumina, San Diego, CA). Next, the sample DNA was denatured using a MiSeq Reagent Kit v3 (Illumina, San Diego, CA), diluted, and subjected to paired-end sequencing ( 75 base pairs) in a MiSeq instrument (Illumina, San Diego, CA). Although the RNA-seq analysis of the sin-3 mutant has been reported previously (Beurton et al. 2019), we analyzed the gene expression profile of this mutant strain to compare the gene expression between the WT and the three corepressor mutants under the same conditions.

\section{RNA-seq data analysis}

The quality of raw sequence data obtained by RNAseq was checked using FastQC software. Trimmomatic software (Bolger et al. 2014) was employed to trim low-quality reads, and the sequence data were mapped to a $C$. elegans reference genome (WormBase Version 261) using HISAT2 software. The count data of WT and mutants were compared using DESeq 2 software (Love et al. 2014) and differentially expressed genes (DEGs; $p$ value $<0.01, \log _{2}$ fold-change; positive or negative) were identified according to a previously described method (Nomoto et al. 2019). To further analyze the DEGs, we identified upregulated genes $\left(\log _{2}\right.$ fold-change $>1$ and $p$ value $<0.01)$ and downregulated genes $\left(\log _{2}\right.$ foldchange $<-1$ and $p$ value $<0.01$ ). Using the DAVID Bioinformatics Resource database (version 6.8) (Dennis et al. 2003), GO enrichment analyses were performed to identify the specific functions of the DEGs.

\section{Results}

\section{hda-1, sin-3, let-418, and spr-1 play a role in C. elegans embryogenesis}

The activities of hda-1, sin-3, let-418, and spr-1 during embryonic development in $C$. elegans were confirmed through determining relative mRNA expression levels and embryonic lethality after either gene knockdown or deletion. Changes in the relative mRNA expression levels of hda-1, let-418, sin-3, and spr-1 in C. elegans during development were analyzed by RT-qPCR at five developmental stages - the early-embryo, middle-embryo, late-embryo, first-larval, and young adult stages. We found that all analyzed genes were expressed throughout embryonic development (Fig. 1). Our results showed that $h d a-l$ expression started at the early embryonic stage in $C$. elegans is consistent with previous results obtained with C. elegans, zebrafish, and mice (Dufourcq et al. 2002; Ma and Schultz 2016; Pillai et al. 2004).

Because $h d a-1($ e1795) mutants are completely sterile (Dufourcq et al. 2002), we used hda-l feeding RNAi to analyze the function of this gene during embryonic development. During C. elegans embryogenesis, the effects of HDA-1 and its corepressors were analyzed by observing the embryonic lethality of $h d a-1(R N A i)$, sin-3(tm1276) deletion, spr-1(ok2144) deletion, and let-418(n3536) temperaturesensitive weak allele. The results show that the embryonic lethality of the let-418(n3536), sin-3(tm1276), and spr1(ok2144) mutants $(10.6 \%, 10.4 \%$, and $5.3 \%$, respectively) was much higher than that of the WT strain (1.1\%) (Fig. 2).

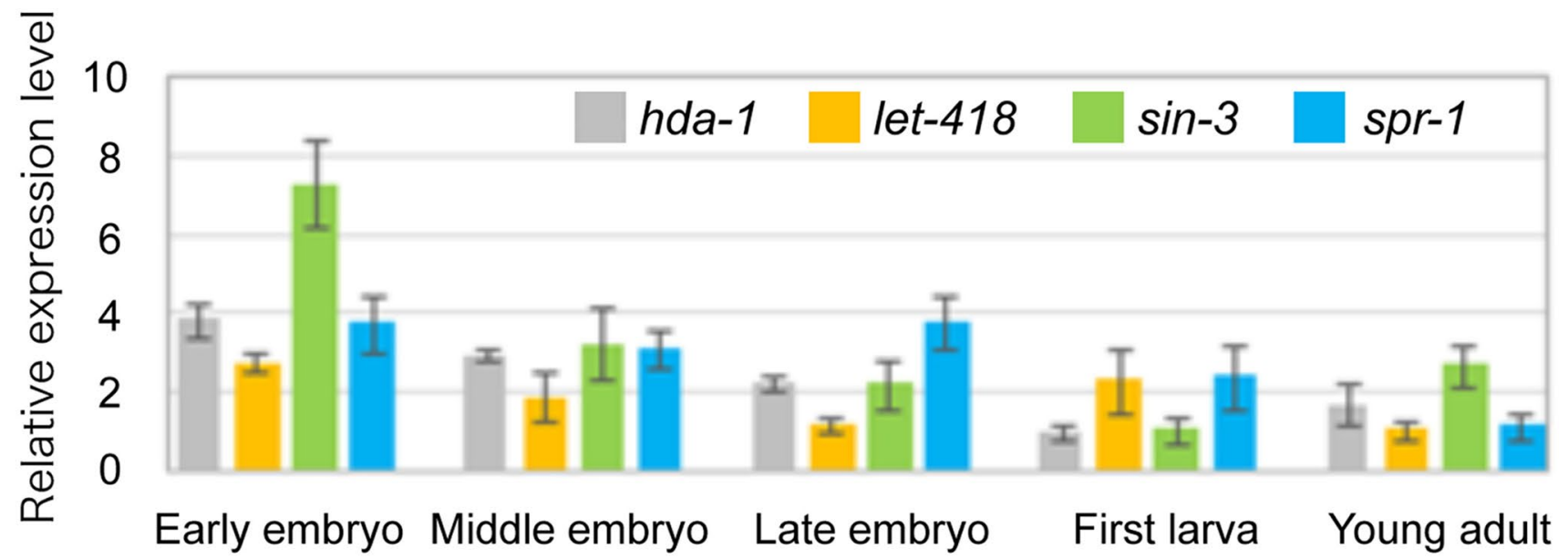

Fig. 1 Comparison of the relative mRNA expression levels of hda1, let-418, sin-3, and spr-1 genes during development. The mRNA expression levels of $h d a-1$, let-418, sin-3, and spr-1 in the earlyembryo, middle-embryo, late-embryo, first-larva, and young-adult stages in WT worms were analyzed by RT-qPCR $(\mathrm{N}=3)$. The
Y45F10D.4 (iron binding protein) gene was used as a reference. The bars and error bars indicate the relative mRNA expression levels and standard deviations, respectively, of hda-1 (gray), let-418 (orange), $\sin -3$ (green), and spr-1 (light blue) at each developmental stage (color figure online) 


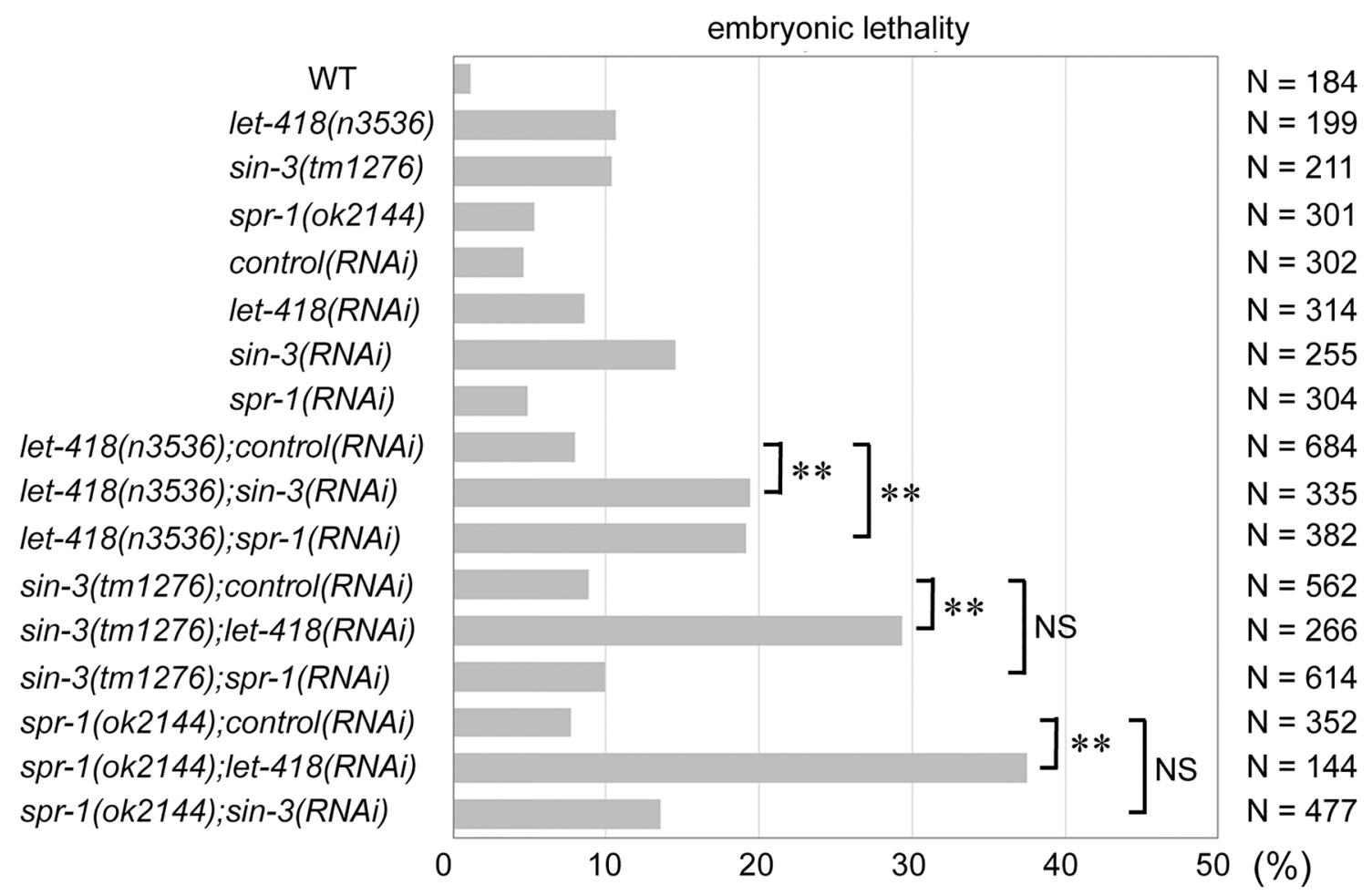

Fig. 2 Genetic interactions among the sin-3, let-418, and spr1 mutants. Embryonic lethality of the WT, let-418(n3536), sin3(tm1276), and spr-1(ok2144) strains (single and double mutants) was analyzed by performing RNAi-based knockdown analysis. Each gray box indicates the embryonic lethality of the WT

Similarly, $h d a-1(R N A i)$ showed higher embryonic lethality (99.7\%, $\mathrm{N}=352$; data not shown) compared to the control (RNAi) (4.6\%) (Fig. 2). The embryonic lethality of hdaI(RNAi) and sin-3(tm1276) mutants was consistent with the findings of previous reports, whereas the embryonic lethality of the let-418(n3536) strain under semi-permissive conditions (maintained at $20^{\circ} \mathrm{C}$ ) was inconsistent (Beurton et al. 2019; Shi and Mello 1998; Turcotte et al. 2018). Overall, relative mRNA expression levels and embryonic lethality rates indicate that $h d a-1, \sin -3$, let- 418 , and $s p r-1$ play a role in C. elegans embryogenesis.

Additionally, we observed the terminal phenotypes of knockdown and mutant embryos using a differential-interference contrast microscope. Similar to that in $h d a-1(R N A i)$ embryos, which was described previously (Shi and Mello 1998), the development of most embryonic-lethal embryos stopped between the ventral cleft-enclosure stage and the threefold stage in the let-418(n3536), sin-3(tm1276), and spr-1(ok2144) mutants (Fig. 3, Table 1). These results indicate that each corepressor is crucial for progression to the middle- and late embryonic developmental stages of $C$. elegans. However, the developmental timing of the embryonic lethality showed no difference. Thus, we could not check the epistatic relationship among the three corepressor strain and the indicated mutant. $p$ values are indicated for Fisher's exact test comparisons with sin-3(tm1276); control(RNAi), let418(n3536);control(RNAi), or spr-1(ok2144);control(RNAi). **p value $<0.01$. NS, not significant. N, number of embryos observed

components because of the similar terminal phenotypes results.

\section{Genetic interactions among let-418, sin-3, and spr-1 during embryonic development suggest possible overlapping roles among these genes}

To identify the functional relationships among $\sin -3$, spr1 , and let-418, we analyzed the genetic interactions among these corepressors by examining the rates of embryonic lethality in strains containing an RNAi-mediated knockdown of a specific gene in a distinct gene deletion background. In an spr-1(ok2144) deletion background and a sin3(tm1276) deletion background, the embryonic lethality of the sin-3(tm1276);spr-1(RNAi) and spr-1(ok2144);sin$3(R N A i)$ mutants $(9.9 \%$ and $13.6 \%$, respectively) was comparable to that of $\sin -3($ tm 1276); control(RNAi) and spr-1(ok2144); control(RNAi) strains $(8.9 \%$ and $7.7 \%$, respectively). These results suggest that $\sin -3$ and $s p r-1$ may have prominent overlapping roles in $C$. elegans during embryogenesis. In contrast, the embryonic lethality of the $\sin$-3(tm1276);let-418(RNAi) and spr-1(ok2144);let-418(RNAi) mutants (29.3\% and $37.5 \%$, respectively) was significantly higher 
Fig. 3 Microscopic images of the terminal phenotypes of let-418, sin-3, and spr-1 mutants with embryonic lethality. Differential-interference contrast micrographs of the let-418(n3536) (a, b), sin3(tm1276) (c, d), and spr1(ok2144) (e, f) mutants. a-f The middle-stage (a, c, e) and late-stage $(\mathbf{b}, \mathbf{d}, \mathbf{f})$ embryos that exhibited a terminal phenotype of embryonic lethality are indicated. The green dotted lines outline the embryos in each panel. White scale bar, $50 \mu \mathrm{m}$ (color figure online)

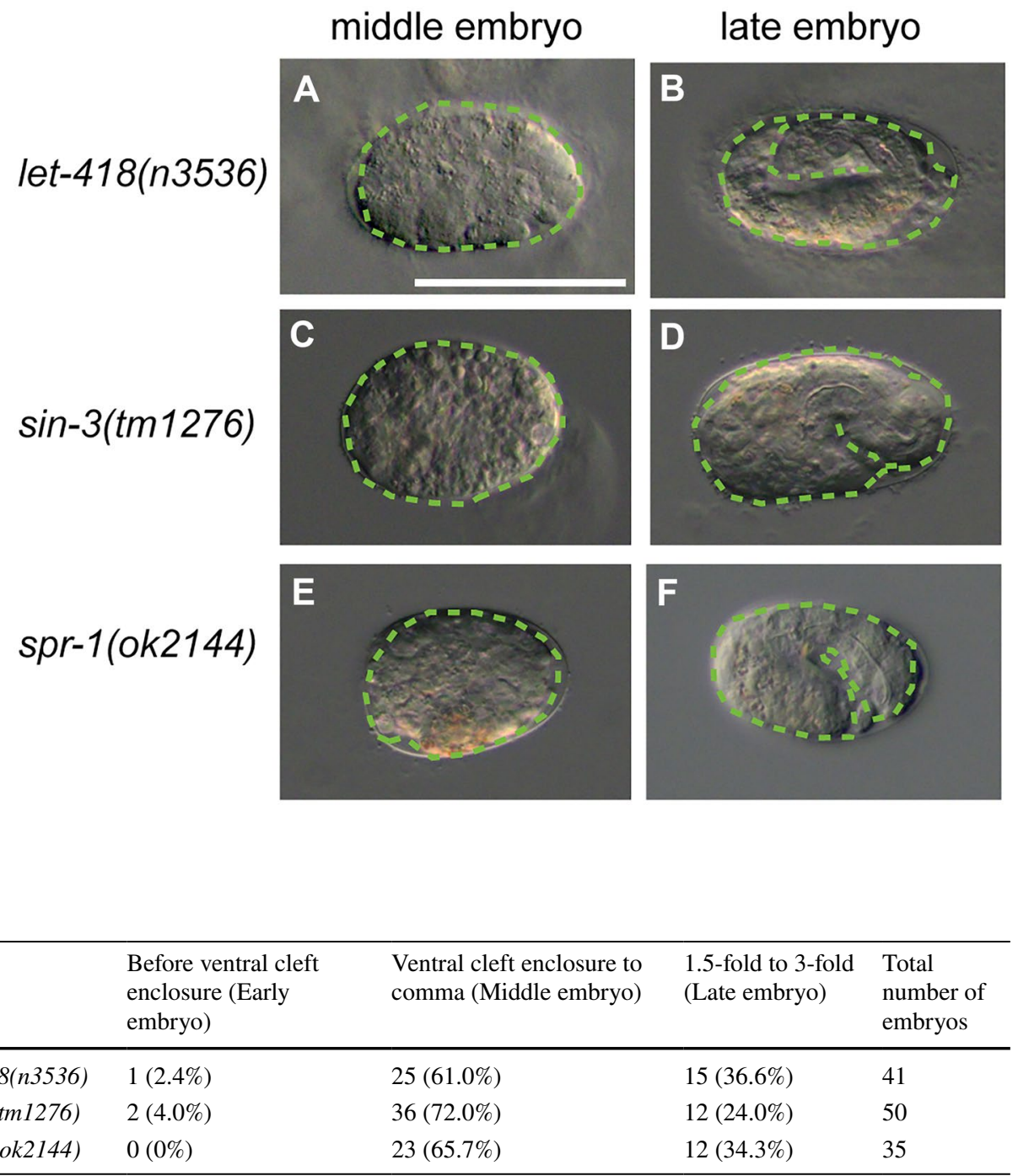

\begin{tabular}{lllll}
\hline & $\begin{array}{l}\text { Before ventral cleft } \\
\text { enclosure (Early } \\
\text { embryo) }\end{array}$ & $\begin{array}{l}\text { Ventral cleft enclosure to } \\
\text { comma (Middle embryo) }\end{array}$ & $\begin{array}{l}\text { 1.5-fold to 3-fold } \\
\text { (Late embryo) }\end{array}$ & $\begin{array}{l}\text { Total } \\
\text { number of } \\
\text { embryos }\end{array}$ \\
\hline let-418(n3536) & $1(2.4 \%)$ & $25(61.0 \%)$ & $15(36.6 \%)$ & 41 \\
$\sin -3($ tm1276) & $2(4.0 \%)$ & $36(72.0 \%)$ & $12(24.0 \%)$ & 50 \\
$\operatorname{spr}-1($ ok2144) & $0(0 \%)$ & $23(65.7 \%)$ & $12(34.3 \%)$ & 35 \\
\hline
\end{tabular}

Table 1 Timing of embryonic lethality in let-418, sin-3, and spr-1 mutants than that of sin-3(tm1276); control(RNAi) and spr1(ok2144); control(RNAi) (8.9\% and $7.7 \%$, respectively) (Fig. 2). These results suggest that let- 418 has a prominent specific role distinct from that of either $\sin -3$ or $s p r-1$. To further evaluate a possible functional relationship among $\sin -3$, spr-1, and let-418, we also examined the rate of embryonic lethality in strains containing RNAi-mediated knockdown of sin-3 and spr-1 in a let-418(n3536) weak allele background. The embryonic lethality of the let418(n3536);sin-3(RNAi) and let-418(n3536);spr-1(RNAi) mutants (19.4\% and $19.1 \%$, respectively) was significantly higher than that of let-418(n3536); control(RNAi) $(8.0 \%)$; these results also suggest that let- 418 has a prominent specific role distinct from that of either sin-3 or spr-1 (Fig. 2). In contrast, the comparable embryonic lethality results of the let-418(n3536); sin-3(RNAi) and let-418(n3536); spr1(RNAi) mutants may also indicate a possible overlapping role among these three genes. Taken together, our genetic interaction analyses suggest a prominent overlapping role among sin-3, spr-1, and let-418 and between sin-3 and spr-1, and a prominent exclusive role of let-418 compared to that of either $\sin -3$ or $\operatorname{spr}$ - 1 .

\section{Analysis of transcriptionally regulated genes in let-418, sin-3, and spr-1 mutants}

To identify genes that were transcriptionally regulated by the three HDAC-1 complexes, we identified groups of genes for which expression levels significantly fluctuated among the corepressor mutants (Fig. 4, Supplementary Fig. 1-3, Supplementary Table 1). Expression-level information for 46,756 transcripts in early WT, let-418(n3536), sin3(tm1276), and spr-1(ok2144) mutant $(\mathrm{N}=3)$ embryos was obtained by performing RNA-seq analysis. Differentially expressed genes (DEGs) in each mutant strain compared to those in the WT were defined as those for which expression 
Fig. 4 Comparison of mRNA expression levels in corepressor mutants by RNA-seq. Venn diagrams showing overlapping upregulated genes (a) and downregulated genes (b) in let-418 embryos (green), sin-3 embryos (blue), spr-1 embryos (red), let-418 and sin-3 embryos (purple), let-418 and spr-1 embryos (yellow), sin-3 and $\operatorname{spr}-1$ embryos (magenta) and let-418, sin-3, and spr-1 embryos (gray) (color figure online)
A

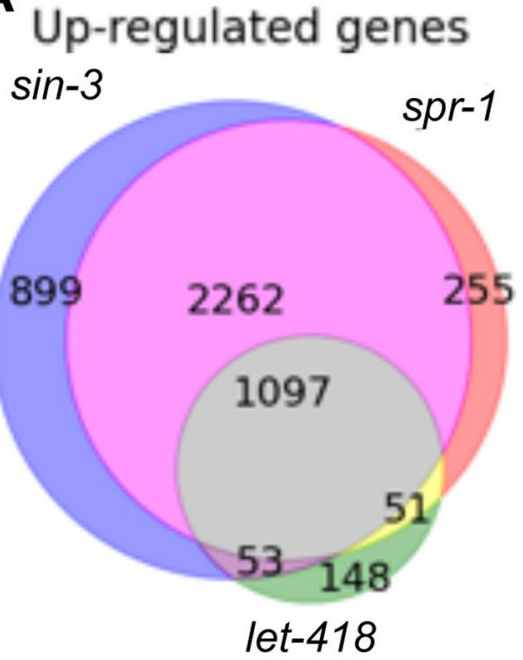

B Down-regulated genes

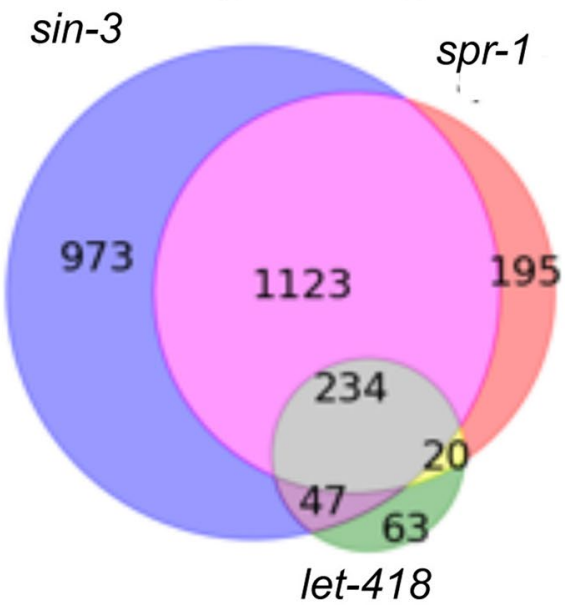

levels were significantly upregulated ( $p$ value $<0.01$ and $\log _{2}$ fold-change $>1$ ) or significantly downregulated ( $p$ value $<0.01$ and $\log _{2}$ fold-change $<1$ ) (Fig. 4). Genes for which expression levels were significantly upregulated or downregulated in the corepressor mutants were defined as transcriptionally repressed and promoted genes, respectively (Supplementary Table. 2).

The transcriptionally repressed genes showed noticeably higher expression levels than the promoted genes in all DEGs results of corepressors, which is consistent with the role of HDAC complexes in negatively regulated transcription. A Venn diagram of the transcriptionally repressed and promoted genes among corepressors showed a similar pattern. Consistent with our observations in genetic interactions analyses, sin-3 and spr- 1 showed highly overlapped targets in both transcriptionally repressed and promoted genes. The overlapped targets between $\sin -3$, spr-1, and let418 were also high, both in the transcriptionally repressed and promoted genes, but less common than the sin-3 and spr-1 shared targets; this indicates that the related repressor complexes of each of the three corepressor components may cooperatively regulate some expression levels or may have compensatory relationships during C. elegans embryogenesis. Considering the utilization of standard bulk RNA-seq analysis in this study, another possibility is that the related repressor complexes of the three corepressor components may have some similar roles, but each repressor complex independently plays the role within a specific cell.

The DEGs were not consistent with our genetic interactions analyses for let- 418 specific targets. The DEG results showed that expression of the let-418 specific targets was noticeably lower, indicating an implausible single prominent role compared to either sin-3 or spr-1. Basically, the fluctuated expression levels recorded in let-418 mutants were less frequent compared to those of either $\sin -3$ or $\operatorname{spr}-1$ mutants. Indeed, usage of the weak loss-of-function let-418 mutants was likely a major factor for the less prominent than expected RNA-seq results and might overshadow the actual outcomes. However, the results from this let- 418 mutant may still reflect the genes strongly related to let-418. Overall, the DEGs results indicate the occurrence of prominent shared roles among sin-3, spr-1, and let-418 and between $\sin -3$ and $s p r-1$, of which the fluctuated genes groups were determined as LET-418-SIN-3-SPR-1 and SIN-3-SPR-1, respectively.

\section{GO enrichment analysis of transcriptionally regulated genes in the LET-418-SIN-3-SPR-1, SIN-3- SPR-1, and LET-418 pathways}

As shown above, our genetic interaction and DEG analyses agree on the shared roles between $\sin -3$ and $s p r-1$ and among sin-3, spr-1, and let-418, but were inconsistent regarding the let-418 specific role. Genetic interactions indicate that let-418 may have prominent unique functions, whereas DEG analysis identified genes with low, fluctuating expression levels that are specific to let-418. To confirm these, we conducted GO enrichment analysis in both transcriptionally repressed and promoted genes for the fluctuating genes groups in LET-418-SIN-3-SPR-1, SIN-3-SPR-1, and the fluctuating genes from let-418(n3536) that did not overlap with other corepressors, determined as LET-418 (Fig. 4, Supplementary Table.3). We highlighted the GO enrichment results related to embryogenic development, cell specification, cell differentiation, cellular function, gene expression, and molecular function. We focused on differences and similarities in GO terms to gain further insight into the overlapping and non-overlapping roles among corepressors in $C$. elegans embryogenesis.

The resulting upregulated genes from each compressor mutant may be directly or indirectly associated with negative 
A

Up-regulated genes (let-418, sin-3, and spr-1)

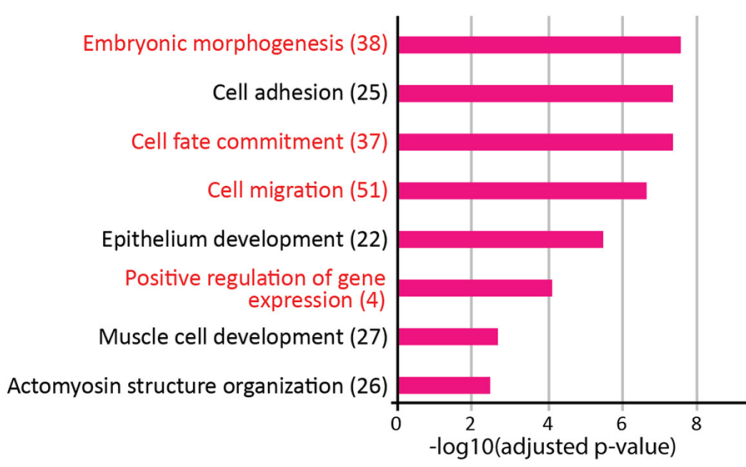

B

Up-regulated genes ( $\sin -3$ and $s p r-1)$

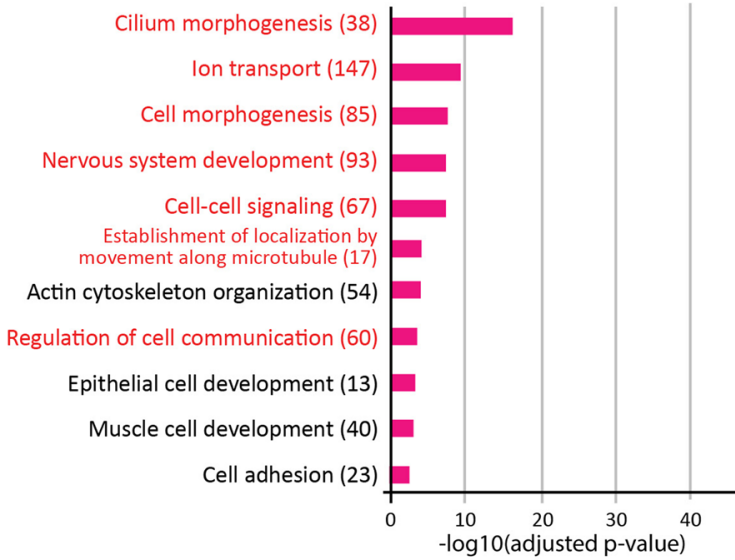

C

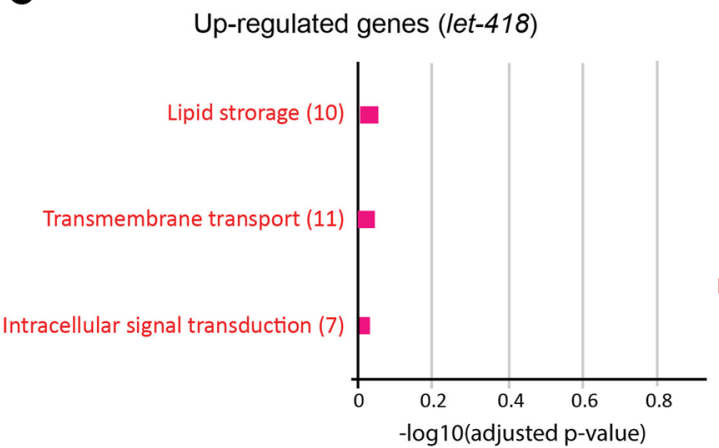

Fig. 5 Gene ontology (GO) analysis of genes dysregulated by the LET-418-SIN-3-SPR-1, SIN-3-SPR-1, and LET-418 pathways. GO terms of the gene groups that were elucidated based on upregulated genes $(\mathbf{a}-\mathbf{c})$ and downregulated genes $(\mathbf{d}-\mathbf{f})$ from the corepressor mutants are indicated. a GO terms associated with genes that were transcriptionally repressed by the LET-418-SIN-3-SPR-1 pathway. b GO terms associated with genes that were transcriptionally repressed by the SIN-3-SPR-1 pathway. c GO terms associated with genes that were transcriptionally repressed by the LET-418 pathway. d GO terms associated with genes that were transcriptionally promoted by the LET-418-SIN-3-SPR-1 pathway. e)GO terms associated with
D

Down-regulated genes (let-418, sin-3, and spr-1)

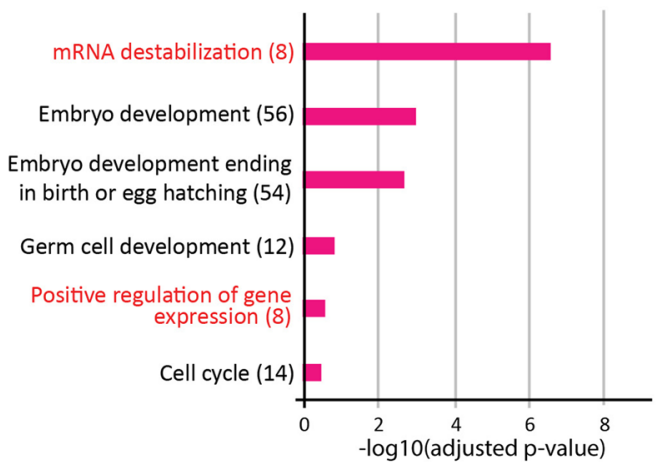

E

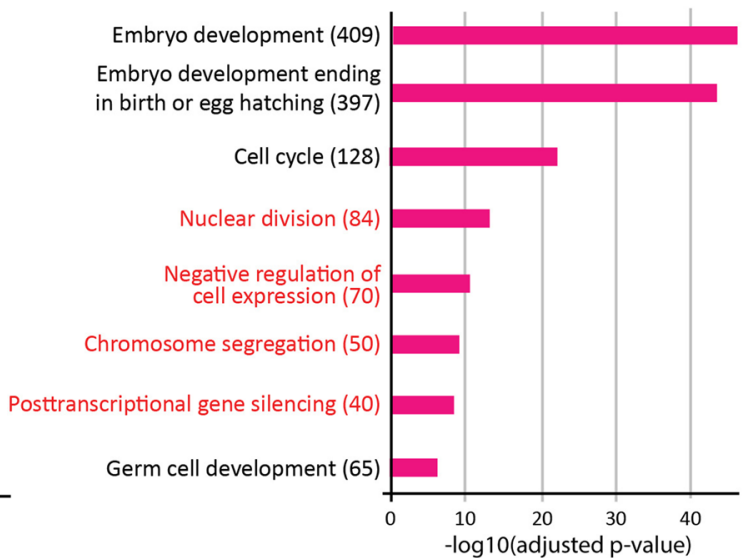

$\mathbf{F}$

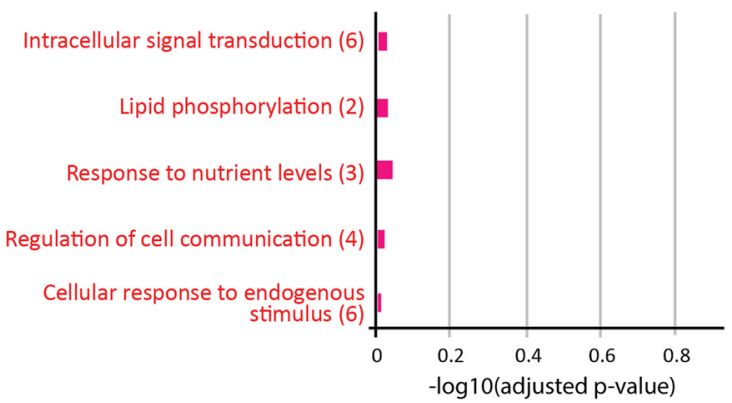

genes that were transcriptionally promoted by the SIN-3-SPR-1 pathway. f GO terms associated with genes that were transcriptionally promoted by the LET-418 pathway. The terms indicated with black text were identified as common GO terms between the LET-418SIN-3-SPR-1 pathway and the SIN-3-SPR-1 pathway. The red text indicates specific GO terms associated with the LET-418-SIN-3SPR-1 pathway, the SIN-3-SPR-1 pathway, and the LET-418 pathway. The $p$ values of the GO terms were determined to evaluate the potential relevance of the associated biological pathways. The numbers in each set of parentheses indicate the numbers of genes that were associated with each GO term (color figure online) 
gene regulation role of each corepressor. The GO enrichment results of LET-418-SIN-3-SPR-1 clearly indicate that the interplay among $\sin -3$, spr-1, and let-418 may be essential to negatively regulate embryonic morphogenesis, cell fate commitment, and positive regulation of gene expression (Fig. 5). The interplay among $\sin -3$, spr-1, and let-418 is also significantly related to cell adhesion, epithelium/epithelial and muscle cells development, and actin cytoskeleton organization, but without let-418, the interplay among $\sin -3$ and spr-1 is still able to regulate these biological processes. In contrast, SIN-3-SPR-1 is specifically associated with numerous biological processes such as cilium morphogenesis, ion transport, cell morphogenesis, nervous system development, cell-cell signaling, establishment of localization along microtubule, and regulation of cell communication (Fig. 5). Although the upregulated genes of LET-418 were associated with GO terms such as lipid storage, transmembrane transport, and intracellular signal transduction, the $p$-values did not reflect statistically significant differences (Fig. 5).

Because the corepressors are mainly associated with the negative regulation of gene expression, the downregulated genes resulting from either corepressor mutant are supposed to be transcriptionally promoted genes caused by repressing activity on other genes (indirectly promoted from repressing activity on other targets). The GO enrichment in the downregulated genes from LET-418-SIN-3-SPR-1 group yielded unique biological processes such as mRNA destabilization and positive regulation of gene expression. Additionally, the GO enrichment in LET-418-SIN-3-SPR-1 had similar results with SIN-3-SPR-1 on embryo development, embryo development ending in birth or egg hatching, germ cell development, and cell cycle, indicating that the interplay between $\sin -3$ and $s p r-1$ with let-418 in these biological processes may be compensated in the absence of let-418. In contrast, the downregulated genes of SIN-3-SPR-1 were significantly associated with nuclear division, negative regulation of gene expression, chromosome segregation, and posttranscriptional gene silencing. Although genes specifically downregulated in the let-418 mutant were associated with GO terms such as cellular response to endogenous stimulus, intracellular signal transduction, response to nutrient levels, lipid phosphorylation, and regulation of cell communication, the $p$-values did not indicate that these associations were statistically significant (Fig. 5). These results imply that relatively few transcripts in let-418(n3536) are affected at a semi-permissive temperature.

We also identified the genes that were significantly upregulated and downregulated in a single mutant of either sin3, spr-1, and let-418 and related to embryogenesis based on the previous GO enrichment results (See Supplementary Fig. 1-3 for the 10 most significantly upregulated and downregulated genes). ECM-related genes were among the most upregulated genes in all three mutants. Genes encoding extracellular matrix (ECM) components, noah-1, lam-2, and lam-3, an ECM receptor, $d g n-1$, and a putative matrix proteinase inhibitor, mig- 6 , were among the most significantly upregulated genes in the let-418(n3536) mutant. In sin-3(tm1276), three ECM genes, noah-1, lam-3, and nid-1, as well as mig-6, were significantly upregulated. In addition, three ECM genes, noah-2, lam-3, and nid-1, and mig-6 were upregulated in the $\operatorname{spr}-1(\mathrm{ok} 2144)$ mutant. In contrast to the upregulated genes, we did not identify any similarly downregulated genes among the three mutants. These results indicate that all three class-I HDAC-1 corepressors significantly repress the expression of ECM-related genes.

\section{Discussion}

During embryogenesis, cells actively undergo division and differentiation by following highly regulated genetic and epigenetic mechanisms. HDAC, a class of epigenetic regulators, catalyzes heterochromatin formation on specific genomic regions by removing acetyl groups from histone tails that results in transcriptional inhibition. The two members of Class-I HDAC, HDAC-1 and -2, are essential for accurate cell division and the pluripotency of embryonic stem (ES) cells. However, only HDAC-1 is essential for controlling ES cell differentiation (Dovey et al. 2010; Jamaladdin et al. 2014). In C. elegans, HDAC-1 is able to form a complex with three distinct corepressor components, SIN-3, SPR-1, and LET-418. HDAC-1 forms the Sin 3 and CoREST complexes while interacting with SIN-3 and SPR-1, respectively (Hayakawa and Nakayama 2011). Furthermore, HDAC1 interaction with LET-418 can form two distinct complexes, MEC and NuRD, depending on the presence of the other components (Passannante et al. 2010). Here, we initially confirmed that $h d a-1, \sin -3, \operatorname{spr}-1$, and let-418 play a role during embryogenesis in C. elegans through mRNA expression, embryonic lethality given either gene knockdown or deletion, and terminal phenotype analyses. Further, our terminal phenotype observations demonstrate that either gene is similarly crucial during the middle- and late embryonic developmental stages. The similar terminal phenotype results, however, complicate the determination of epistatic relationships among these corepressor components. Further studies are required to identify the signal transduction cascades activated by these corepressors in each pathway.

Although HDAC-1 is known to be essential protein during embryogenesis, the functional relationships among its complexes remain unclear. Here, we sought to elucidate those through genetic interactions and DEG analyses. Our results suggest prominent shared roles between $\sin -3$ and $s p r-1$ and among sin-3, spr-1, and let-418. In contrast, genetic interactions indicate let- 418 as having a prominent independent role, whereas DEG analysis of the let-418 mutant identified 
only genes with low, fluctuating gene expression that did not overlap with either sin-3 or $s p r-1$ (Fig. 4). A possible explanation for these results is that the repressor complex involving LET-418 may be regulated by another repressor complex or may frequently function dependent on another repressor complex. However, it should be noted that the let418 mutant used in this study is a weak allele of let-418, and, therefore, the transcriptome analysis may not fully reflect the normal function of this gene during embryonic development.

Comparative GO analysis of the fluctuated genes among the three specified groups, LET-418-SIN-3-SPR1, SIN-3-SPR-1, and LET-418, indicates that similar GO terms were enriched between the LET-418-SIN-3-SPR-1 group and the SIN-3-SPR-1 group but not the LET-418 group. Further analyses of the GO terms indicate that many of the suppressed genes are related to neuronal, epithelial, and muscle development and actin-structure regulation. Enhanced expression of genes related to embryonic and germ cell development and cell cycle progression was identified as a common feature between the fluctuated genes in LET-418-SIN-3-SPR-1 and SIN-3-SPR-1 groups (Fig. 6). There are several possibilities that can explain the overlapping fluctuating genes either in LET418-SIN-3-SPR-1 group or SIN-3-SPR-1 group. First, the related repressor complexes may cooperatively regulate some expression levels or may have compensatory relationships. This possibility implies that the cooperative regulation of gene expression among the related repressor complex is important for the precise regulation during embryonic development. Second, the related repressor complexes may have similar functions but each repressor complex independently plays a role within a specific cell. Further investigation is needed to clarify this issue.

GO enrichment analysis of the repressed genes in the SIN-3-SPR-1 group indicates that they are related to cell morphogenesis, intracellular communication, and microtubule-related transport (Fig. 6). In contrast, the promoted genes of this group are related to nuclear-related cell division and negative regulation of gene expression (Fig. 6). How the SIN-3-SPR-1 group regulates these biological functions? Interestingly, mSin $3 \mathrm{~A}$ and CoREST are co-expressed in mouse embryos at E11.5, and

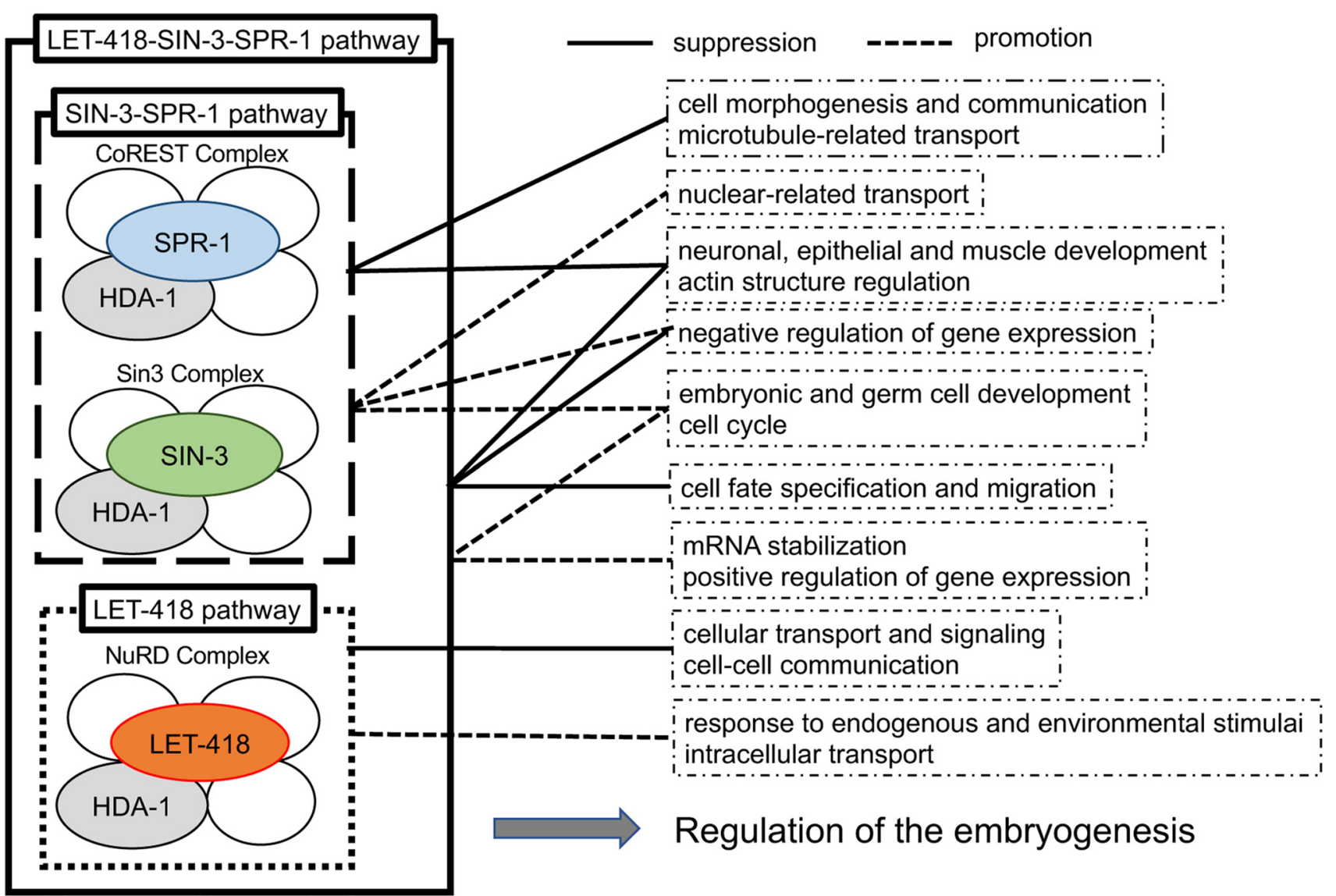

Fig. 6 Model of the functional relationships among the LET-418SIN-3-SPR-1, SIN-3-SPR-1, and LET-418 pathways. The LET-418SIN-3-SPR-1, SIN-3-SPR-1, and LET-418 pathways positively and negatively regulate common and pathway-specific biological functions to influence embryonic development 
mSin3A has been shown to act as a functional component of the REST-CoREST suppressor complex (Grimes et al. 2000). Thus, negative transcriptional regulation of the SIN-3-CoREST suppressor complex may be conserved in both vertebrates and invertebrates.

GO enrichment analysis indicates that the repressed genes in LET-418-SIN-3-SPR-1 group are related to cell morphogenesis, cell fate specification, cell migration, and negative regulation of gene expression (Fig. 6). Although $h d a-1$ has been shown to positively regulate neuronal and distal-tip cell migration during post-embryonic development in C. elegans (Dufourcq et al. 2002; Zinovyeva et al. 2006), here we show that the related repressor complexes of LET-418, SIN-3, SPR-1 likely modulate the negative regulation of cell migration during embryonic development. In contrast, the promoted genes in LET-418-SIN-3-SPR-1 group are related to mRNA stabilization and positive regulation of gene expression. Differences in gene expression and mRNA stabilization occur in somatic and germ cell linages throughout embryonic development in C. elegans, which might reflect the function of LET-418, SIN-3, and SPR-1. Taken together, our results indicate that the three corepressor components positively and negatively regulate cell type-specific functions during embryogenesis. However, the exact mechanism of the LET-418-, SIN-3-, and SPR1-related repressor complexes in negatively regulating cell differentiation and movement, promoting cell type-specific gene stabilization, and positively and negatively regulating the expression levels of different genes remains unknown.

The repressed genes in the LET-418-specific group are related to the control of cellular transport and signaling. In contrast, the promoted genes are related to controlling cell-cell communication, cellular responses to endogenous and environmental stimuli, and intracellular transport (Fig. 6). Our results are inconsistent with previous studies on LET-418 function during embryogenesis, which are related to the prevention of germline development and repressing the neuronal fate during embryonic development (KäserPébernard et al. 2016; Unhavaithaya et al. 2002). We used a weak allele of the let-418 mutant, and further studies with a strong loss-of-function let-418 mutant are required to confirm its normal cellular roles.

We also highlighted several mostly fluctuating genes in DEG analyses (Supplementary Fig. 1-3). Similar to previous findings indicating that ECM genes are upregulated in hda-1(RNAi) embryos (Whetstine et al. 2005), we found that genes encoding ECM- and ECM-related mRNAs are significantly upregulated in the C. elegans HDAC- 1 corepressor mutants, specifically let-418(n3536), sin-3(tm1276), and spr-1(ok2144). The upregulated ECM genes (noah-1, noah2, nid-1, lam-2, and lam-3) and ECM-related genes (dgn-1 and mig-6) play important roles in embryonic morphogenesis or neuronal patterning (Huang et al. 2003; Johnson et al. 2006; Kao et al. 2006; Kawano et al. 2009; Kim and Wadsworth 2000; Vuong-Brender et al. 2017), and, therefore, their temporal suppression is important for the regulation of embryonic development. Thus, we speculate that all three corepressors serve a common role that is required for the negative regulation of genes encoding ECM- and ECMrelated proteins.

\section{Conclusions}

Using combined analyses of genetic interactions and transcriptome levels, we identified the overlapping functions among the $C$. elegans homologs of the HDAC-1 corepressors, LET-418, SIN-3, and SPR-1. Our genetic interaction and DEG analyses were consistent regarding the shared roles between sin-3 and spr-1 and among sin-3, spr-1, and let-418, but inconsistent regarding the let-418-specific role. Our terminal phenotype analyses show that sin-3, spr-1, and let-418 are crucial for the progression to the middle- and late embryonic developmental stages of $C$. elegans, which is similar to previously described $h d a-l(R N A i)$ embryos. Finally, comparative RNA-seq analysis of these three corepressor components indicates that approximately half of upregulated and downregulated genes were associated with the SIN-3-SPR-1 group. Similarly, $10-20 \%$ of the upregulated and downregulated genes were associated with the LET-418-SIN-3-SPR-1 group. Taken together, our findings suggest that the classI HDAC-1 corepressors, LET-418, SIN-3, and SPR-1 may cooperatively regulate the expression levels of some genes during C. elegans embryogenesis, or may have some similar roles but function independently within a specific cell.

Supplementary Information The online version contains supplementary material available at https://doi.org/10.1007/s13258-021-01076-1.

Acknowledgements We thank Mr. Yusuke Nomoto and Mr. Takahiro Nakamura for their support and helpful comments. Some strains were provided by the CGC, which is funded by the NIH Office of Research Infrastructure Programs (Grant Number P40 OD010440), the C. elegans Gene Knockout Consortium, and the National Bioresource Project in Japan (lead by S. Mitani). This research was funded by the MEXT-Supported Program for the Strategic Research Foundation at Private Universities, Grant Number S1511028, and the Takeda Science Foundation.

Authors' contributions MI, YK, YO, and TH conceived the project. MI supervised the study. YK, YO, TH, NO, and AE collected the samples and worked on phenotypes in the field. YO constructed RNA-seq libraries and carried out sequencing. YO performed the qRT-PCR. YK, YO, GY, HK, and MF performed the bioinformatics and data analysis. YK, $\mathrm{TH}, \mathrm{NO}$, and AE constructed the Feeding RNAi vector, performed transgenic experiment and phenotyping. MI, YK, and YO drafted the manuscript. MI, YK, YO, and MF revised the manuscript. All authors read and approved the final manuscript. 
Data availability All data and samples described in this study will be freely provided upon request.

\section{Declaration}

Conflict of interest The authors have declared no competing interests.

Open Access This article is licensed under a Creative Commons Attribution 4.0 International License, which permits use, sharing, adaptation, distribution and reproduction in any medium or format, as long as you give appropriate credit to the original author(s) and the source, provide a link to the Creative Commons licence, and indicate if changes were made. The images or other third party material in this article are included in the article's Creative Commons licence, unless indicated otherwise in a credit line to the material. If material is not included in the article's Creative Commons licence and your intended use is not permitted by statutory regulation or exceeds the permitted use, you will need to obtain permission directly from the copyright holder. To view a copy of this licence, visit http://creativecommons.org/licenses/by/4.0/.

Open Access This article is licensed under a Creative Commons Attribution 4.0 International License, which permits use, sharing, adaptation, distribution and reproduction in any medium or format, as long as you give appropriate credit to the original author(s) and the source, provide a link to the Creative Commons licence, and indicate if changes were made. The images or other third party material in this article are included in the article's Creative Commons licence, unless indicated otherwise in a credit line to the material. If material is not included in the article's Creative Commons licence and your intended use is not permitted by statutory regulation or exceeds the permitted use, you will need to obtain permission directly from the copyright holder. To view a copy of this licence, visit http://creativecommons.org/licenses/by/4.0/.

\section{References}

Bender AM, Kirienko NV, Olson SK, Esko JD, Fay DS (2007) lin-35/ $\mathrm{Rb}$ and the CoREST ortholog spr-1 coordinately regulate vulval morphogenesis and gonad development in C. elegans. Dev Biol 302:448-462

Beurton F, Stempor P, Caron M, Appert A, Dong Y, Chen RA, Cluet D, Couté Y, Herbette M, Huang N et al (2019) Physical and functional interaction between SET1/COMPASS complex component CFP-1 and a Sin3S HDAC complex in C. elegans. Nucleic Acids Res 47:11164-11180

Bolger AM, Lohse M, Usadel B (2014) Trimmomatic: a flexible trimmer for Illumina sequence data. Bioinformatics 30:2114-2120

Brenner S (1974) The genetics of Caenorhabditis elegans. Genetics 77:71-94

Cavalli G (2006) Chromatin and epigenetics in development: blending cellular memory with cell fate plasticity. Development 133:2089-2094

Ceol CJ, Stegmeier F, Harrison MM, Horvitz HR (2006) Identification and classification of genes that act antagonistically to let-60 Ras signaling in Caenorhabditis elegans vulval development. Genetics 173:709-726

Choy SW, Wong YM, Ho SH, Chow KL (2007) C. elegans SIN-3 and its associated HDAC corepressor complex act as mediators of male sensory ray development. Biochem Biophys Res Commun 358:802-807

Cosgrove MS, Boeke JD, Wolberger C (2004) Regulated nucleosome mobility and the histone code. Nat Struct Mol Biol 11:1037-1043
Dennis G, Sherman BT, Hosack DA, Yang J, Gao W, Lane HC, Lempicki RA (2003) DAVID: database for annotation, visualization, and integrated discovery. Genome Biol 4:R60

Dovey OM, Foster CT, Cowley SM (2010) Histone deacetylase 1 (HDAC1), but not HDAC2, controls embryonic stem cell differentiation. Proc Natl Acad Sci 107:8242-8247

Dufourcq P, Victor M, Gay F, Calvo D, Hodgkin J, Shi Y (2002) Functional requirement for histone deacetylase 1 in Caenorhabditis elegans gonadogenesis. Mol Cell Biol 22:3024-3034

Feng D, Liu T, Sun Z, Bugge A, Mullican SE, Alenghat T, Liu XS, Lazar MA (2011) A circadian rhythm orchestrated by histone deacetylase 3 controls hepatic lipid metabolism. Science 331:1315-1319

Garcia-Ramirez M, Rocchini C, Ausio J (1995) Modulation of chromatin folding by histone acetylation. J Biol Chem 270:17923-17928

Genome sequence of the nematode C. elegans: a platform for investigating biology. (1998) Science 282:2012-2018

Grimes JA, Nielsen SJ, Battaglioli E, Miska EA, Speh JC, Berry DL, Atouf F, Holdener BC, Mandel G, Kouzarides T (2000) The corepressor $\mathrm{mSin} 3 \mathrm{~A}$ is a functional component of the REST-CoREST repressor complex. J Biol Chem 275:9461-9467

Hayakawa T, Nakayama J (2011) Physiological roles of class I HDAC complex and histone demethylase. J Biomed Biotechnol 2011:129383

Hoogewijs D, Houthoofd K, Matthijssens F, Vandesompele J, Vanfleteren JR (2008) Selection and validation of a set of reliable reference genes for quantitative sod gene expression analysis in C. elegans. BMC Mol Biol 9:9

Huang CC, Hall DH, Hedgecock EM, Kao G, Karantza V, Vogel BE, Hutter H, Chisholm AD, Yurchenco PD, Wadsworth WG (2003) Laminin alpha subunits and their role in C. elegans development. Development 130:3343-3358

Jamaladdin S, Kelly RDW, O'Regan L, Dovey OM, Hodson GE, Millard CJ, Portolano N, Fry AM, Schwabe JWR, Cowley SM (2014) Histone deacetylase (HDAC) 1 and 2 are essential for accurate cell division and the pluripotency of embryonic stem cells. Proc Natl Acad Sci 111:9840-9845

Johnson RP, Kang SH, Kramer JM (2006) C. elegans dystroglycan DGN-1 functions in epithelia and neurons, but not muscle, and independently of dystrophin. Development 133:1911-1921

Kamath RS, Martinez-Campos M, Zipperlen P, Fraser AG, Ahringer J (2001) Effectiveness of specific RNA-mediated interference through ingested double-stranded RNA in Caenorhabditis elegans. Genome Biol 2:Research0002

Kao G, Huang CC, Hedgecock EM, Hall DH, Wadsworth WG (2006) The role of the laminin beta subunit in laminin heterotrimer assembly and basement membrane function and development in C. elegans. Dev Biol 290:211-219

Käser-Pébernard S, Pfefferli C, Aschinger C, Wicky C (2016) Finetuning of chromatin composition and Polycomb recruitment by two Mi2 homologues during C. elegans early embryonic development. Epigenetics Chromatin 9:39

Kawano T, Zheng H, Merz DC, Kohara Y, Tamai KK, Nishiwaki $\mathrm{K}$, Culotti JG (2009) C. elegans mig-6 encodes papilin isoforms that affect distinct aspects of DTC migration, and interacts genetically with mig-17 and collagen IV. Development 136:1433-1442

Kim S, Wadsworth WG (2000) Positioning of longitudinal nerves in C. elegans by nidogen. Science $288: 150-154$

Knutson SK, Chyla BJ, Amann JM, Bhaskara S, Huppert SS, Hiebert SW (2008) Liver-specific deletion of histone deacetylase 3 disrupts metabolic transcriptional networks. Embo j 27:1017-1028

Love MI, Huber W, Anders S (2014) Moderated estimation of fold change and dispersion for RNA-seq data with DESeq2. Genome Biol 15:550 
Ma P, Schultz RM (2016) HDAC1 and HDAC2 in mouse oocytes and preimplantation embryos: specificity versus compensation. Cell Death Differ 23:1119-1127

Mannervik M, Levine M (1999) The Rpd3 histone deacetylase is required for segmentation of the Drosophila embryo. Proc Natl Acad Sci USA 96:6797-6801

Miller KM, Tjeertes JV, Coates J, Legube G, Polo SE, Britton S, Jackson SP (2010) Human HDAC1 and HDAC2 function in the DNAdamage response to promote DNA nonhomologous end-joining. Nat Struct Mol Biol 17:1144-1151

Montgomery RL, Davis CA, Potthoff MJ, Haberland M, Fielitz J, Qi X, Hill JA, Richardson JA, Olson EN (2007) Histone deacetylases 1 and 2 redundantly regulate cardiac morphogenesis, growth, and contractility. Genes Dev 21:1790-1802

Nomoto Y, Kubota Y, Ohnishi Y, Kasahara K, Tomita A, Oshime T, Yamashita H, Fahmi M, Ito M (2019) Gene Cascade Finder: a tool for identification of gene cascades and its application in Caenorhabditis elegans. PLoS ONE 14:e0215187

Passannante M, Marti CO, Pfefferli C, Moroni PS, Kaeser-Pebernard S, Puoti A, Hunziker P, Wicky C, Müller F (2010) Different Mi-2 complexes for various developmental functions in Caenorhabditis elegans. PLoS ONE 5:e13681

Pillai R, Coverdale LE, Dubey G, Martin CC (2004) Histone deacetylase 1 (HDAC-1) required for the normal formation of craniofacial cartilage and pectoral fins of the zebrafish. Dev Dyn 231:647-654

Ranawade AV, Cumbo P, Gupta BP (2013) Caenorhabditis elegans histone deacetylase hda-1 is required for morphogenesis of the vulva and LIN-12/Notch-mediated specification of uterine cell fates. G3 (Bethesda) 3:1363-1374

Saudenova M, Wicky C (2018) The Chromatin Remodeler LET-418/ Mi2 is Required Cell Non-Autonomously for the Post-Embryonic Development of Caenorhabditis elegans. J Dev Biol 7

Shi Y, Mello C (1998) A CBP/p300 homolog specifies multiple differentiation pathways in Caenorhabditis elegans. Genes Dev 12:943-955

Solari F, Ahringer J (2000) NURD-complex genes antagonise Rasinduced vulval development in Caenorhabditis elegans. Curr Biol 10:223-226

Sulston JE, Schierenberg E, White JG, Thomson JN (1983) The embryonic cell lineage of the nematode Caenorhabditis elegans. Dev Biol 100:64-119

Sun Z, Miller RA, Patel RT, Chen J, Dhir R, Wang H, Zhang D, Graham MJ, Unterman TG, Shulman GI et al (2012) Hepatic Hdac3 promotes gluconeogenesis by repressing lipid synthesis and sequestration. Nat Med 18:934-942
Turcotte CA, Sloat SA, Rigothi JA, Rosenkranse E, Northrup AL, Andrews NP, Checchi PM (2018) Maintenance of Genome Integrity by Mi2 Homologs CHD-3 and LET-418 in Caenorhabditis elegans. Genetics 208:991-1007

Unhavaithaya Y, Shin TH, Miliaras N, Lee J, Oyama T, Mello CC (2002) MEP-1 and a homolog of the NURD complex component Mi-2 Act together to maintain germline-soma distinctions in $C$. elegans. Cell 111:991-1002

Vaquero A, Sternglanz R, Reinberg D (2007) NAD+-dependent deacetylation of $\mathrm{H} 4$ lysine 16 by class III HDACs. Oncogene 26:5505-5520

Vecera J, Bartova E, Krejci J, Legartova S, Komurkova D, Ruda-Kucerova J, Stark T, Drazanova E, Kasparek T, Sulcova A et al (2018) HDAC1 and HDAC3 underlie dynamic H3K9 acetylation during embryonic neurogenesis and in schizophrenia-like animals. J Cell Physiol 233:530-548

von Zelewsky T, Palladino F, Brunschwig K, Tobler H, Hajnal A, Muller F (2000) The C. elegans Mi-2 chromatin-remodelling proteins function in vulval cell fate determination. Development 127:5277-5284

Vuong-Brender TTK, Suman SK, Labouesse M (2017) The apical ECM preserves embryonic integrity and distributes mechanical stress during morphogenesis. Development 144:4336-4349

Wenzel D, Palladino F, Jedrusik-Bode M (2011) Epigenetics in $C$. elegans: facts and challenges. Genesis 49:647-661

Whetstine JR, Ceron J, Ladd B, Dufourcq P, Reinke V, Shi Y (2005) Regulation of tissue-specific and extracellular matrix-related genes by a class I histone deacetylase. Mol Cell 18:483-490

Wilting RH, Yanover E, Heideman MR, Jacobs H, Horner J, van der Torre J, DePinho RA, Dannenberg JH (2010) Overlapping functions of Hdac1 and Hdac2 in cell cycle regulation and haematopoiesis. Embo J 29:2586-2597

Yang XJ, Seto E (2008) The Rpd3/Hda1 family of lysine deacetylases: from bacteria and yeast to mice and men. Nat Rev Mol Cell Biol 9:206-218

Zinovyeva AY, Graham SM, Cloud VJ, Forrester WC (2006) The $C$. elegans histone deacetylase HDA-1 is required for cell migration and axon pathfinding. Dev Biol 289:229-242

Publisher's Note Springer Nature remains neutral with regard to jurisdictional claims in published maps and institutional affiliations. 